\title{
Der digitale Zwilling in der endovaskulären Versorgung
}

\author{
André Hemmler ${ }^{1} \cdot$ Christian Reeps $^{2} \cdot$ Brigitta Lutz $^{2} \cdot$ Michael W. Gee ${ }^{1,}{ }^{*}$ \\ ${ }^{1}$ Mechanics \& High Performance Computing Group, Technische Universität München, \\ Parkring 35, 85748 Garching b. München, Germany \\ 2 Universitätsklinikum Carl Gustav Carus, Fetscherstraße 74, 01307 Dresden, Germany \\ ${ }^{*}$ Korrespondenzautor: E-Mail: gee@tum.de
}

\section{Zusammenfassung}

Hintergrund: Die endovaskuläre Behandlung (EVAR) von abdominalen Aortenaneurysmen (AAA) erfordert einen komplexen und bisher stark erfahrungsbasierten präoperativen Planungsprozess z. B. bzgl. Stentgrafttypen Auswahl und Oversizing, welcher an die individuellen patientenspezifischen Gegebenheiten angepasst sein muss.

Fragestellung: Ob die Verwendung eines virtuellen digitalen Zwillings potentiell hilfreich sein kann diesen erfahrungsbasierten Planungsprozess $\mathrm{zu}$ objektivieren und $\mathrm{zu}$ optimieren, um damit das methodenassoziierte Komplikationsrisiko zu senken, soll in der vorliegenden Arbeit untersucht werden.

Methoden: Basierend auf präoperativen patientenspezifischen Daten werden wirklichkeitsgetreue AAA sowie wirklichkeitsgetreue Stentgraft Simulationsmodelle gängiger kommerzieller Stentgrafts erzeugt. Anschließend wird eine virtuelle endovaskuläre AAA Reparatur zur Vorhersage der postoperativen Konfiguration von Stentgraft und AAA verwendet. Unterschiedliche Anwendungsbeispiele dieser Prozesskette sollen den potentiellen Nutzen eines digitalen Zwillings in der endovaskulären Therapie aufzeigen.

Ergebnisse: Die potentielle Anwendbarkeit und der Nutzen eines digitalen Zwillings zur Optimierung der präoperativen Stentgraft Auswahl und Größenbestimmung sowie zur prädiktiven Einschätzung der Komplikationswahrscheinlichkeit basierend auf mechanischen und geometrischen Kenngrößen konnten exemplarisch demonstriert und validifiziert werden.

Schlussfolgerungen: Die gute Vorhersagegüte macht den digitalen Zwilling zu einem vielversprechenden Planungswerkzeug in der präoperativen Planungsphase der endovaskulären AAA Versorgung mit denkbar vielseitigen Anwendungsmöglichkeiten.

\section{Schlüsselwörter}

abdominales Aortenaneurysma, endovaskuläre Aneurysmareparatur, Stentgraft, personalisierte Medizin, digitaler Zwilling 


\title{
The digital twin in the endovascular repair
}

\begin{abstract}
Background: Endovascular aortic repair (EVAR) of abdominal aortic aneurysms (AAA) requires a very complex preoperative planning process, e.g. with respect to stent-graft selection and stent-graft oversizing, which must be individually adapted to the patientspecific case and which is strongly based on the interventionalist's experience.

Objectives: In this study, it is investigated whether the use of a digital twin could increase objectivity and optimize the experience based preoperative planning process and hence reduce the method-associated complication rate.

Methods: Based on preoperative patient-specific data, realistic AAA and realistic stentgraft simulation models of common commercial stent-grafts are generated. Subsequently, virtual endovascular AAA repair is used to predict the postoperative configuration of stent-graft and AAA. Different application examples of this process chain are intended to demonstrate the potential benefits of the use of a digital twin in EVAR.

Results: The potential applicability and the benefit of a digital twin for the optimization of the preoperative stent-graft selection and sizing as well as for the predictive estimation of the complication likelihood based on mechanical and geometrical parameters could be exemplarily demonstrated and validated.

Conclusions: The good predictive quality makes the digital twin a highly promising planning tool in the preoperative planning phase of EVAR with potentially versatile applications.
\end{abstract}

\section{Keywords}

abdominal aortic aneurysm, endovascular repair, stent-graft, personalized medicine, digital twin

\section{Hinführung zum Thema}

Die endovaskuläre Therapie (EVAR) hat sich in den letzten Jahren als Standardmethode zur Behandlung eines abdominalen Aortenaneurysmas etabliert. Methodenassoziierte Komplikationen sowie ein komplexer, stark erfahrungsbasierter präoperativer Planungsprozess bleiben allerdings große Herausforderungen der endovaskulären Therapie. Virtuelle Stentgraftimplantationen mittels digitalem Zwilling könnten dabei den präoperativen Entscheidungsprozess z. B. bzgl. Stentgraft Auswahl und Stentgraft Oversizing optimieren und langfristig das methodenassoziierte Komplikationsrisiko der endovaskulären Therapie senken. 


\section{Einleitung}

\section{Endovaskuläre AAA Versorgung}

Aufgrund Ihrer deutlich geringeren operativen Belastung, ihrer überlegenen Frühergebnisse sowie deutlicher technischer Verbesserungen hat sich die endovaskuläre Therapie als Standardmethode zur Behandlung eines Aortenaneurysmas in der Versorgungsrealität durchgesetzt. Mittlerweile werden deshalb in der BRD weit mehr als 70\% aller infrarenalen AAA minimal-invasiv mittels Standard-EVAR versorgt [10]. Zusätzliche „Neuerungen“ wie fenestrierte und gebranchte Stentprothesen oder die Chimney (Parallel-Graft) Technik erlauben zudem eine Behandlung auch komplexester Aneurysmapathologien mit Einbeziehung der reno-viszeralen Manschette oder der Iliacalbifurkationen, sodass mittlerweile rein technisch gesehen in spezialisierten Zentren mehr als 90\% aller AAA endovaskulär versorgt werden können.

\section{Methodenassoziierte Komplikationen der endovaskulären AAA Versorgung}

Hauptprobleme der endovaskulären AAA Therapie sind, im Vergleich zur offenoperativen AAA Sanierung, jedoch die wesentlich häufigeren methodenassoziierten Spätkomplikationen nach EVAR im Langzeitverlauf. Diese zwingen zu häufigen klinischen Nachkontrollen bzw. Sekundärinterventionen und wiegen letztendlich die hervorragenden Frühergebnisse auf. $\mathrm{Zu}$ nennen sind hier insbesondere vermeidbare oder auch später im Verlauf auftretende primäre und sekundäre Typ I und III Endoleckagen bzw. Prothesenschenkelverschlüsse, welche durch Sekundärdilationen im Bereich der proximalen und distalen Landungszonen, Stentgraftmigration, Dislokationen und Komponentenseparation entstehen und im schlimmsten Falle zu einem Therapieversagen mit Sekundärruptur bzw. schwerer Extremitätenischämie führen können. Vor allem schwierige Aneurysmageometrien außerhalb der Herstellerempfehlungen (sog. IFU, engl. "Instructions for use") mit z. B. ausgeprägtem Aneurysma Kinking zeigen eine drastisch erhöhte Sekundärkomplikationsrate [17]. Andererseits stehen dem endovaskulären Aortenspezialisten heutzutage eine Vielzahl von modernen mechanisch und konzeptionell unterschiedlichen Stentprothesensystemen, patientenspezifische Spezialprothesen sowie weiter flankierende Maßnahmen zur Verfügung, um auch komplexeste Aneurysmageometrien initial erfolgreich versorgen zu können. Die basalen und damit für den kurz und langfristigen Implantationserfolg essentiellen Entscheidungsprozesse bezüglich optimaler Stentgrafttypenauswahl, Oversizing in konischen, angulierten und sanduhrförmigen Hälsen, Längenauswahl in gekinkten Geometrien oder Vorhersage der intraoperativen Implantationsgeometrie bei angulierten Hälsen sind jedoch bislang ausschließlich erfahrungsbasiert.

Realitätsnahe virtuelle Stentgraftimplantationen mittels digitalem Zwilling könnten hier zukünftig helfen solche präoperativen Entscheidungsprozesse zu objektivieren bzw. präoperativ eine Risikostratifizierung für sekundär Komplikationen vorzunehmen.

\section{Potentielle Anwendungen des digitalen Zwillings in der endovaskulären AAA Versorgung}

Trotz regelmäßiger Nachuntersuchungen mittels CT oder Ultraschall im Fall der endovaskulären Versorgung lassen sich an der realen Einheit (= implantierter Stentgraft), dem sogenannten realen Zwilling, nur sehr limitierte Kenngrößen ermitteln, wie z. B. die postoperative Lage und geometrische Konfiguration des Stentgrafts, 
Attachement- und Überlappungszonen. Der digitale Zwilling, als digitale Nachbildung dieser realen Einheit [5], kann hier potentiell zusätzliche Daten und Informationen liefern, welche an der realen Einheit nicht oder nur sehr mühsam ermittelt werden können aber wesentlich über den langfristigen Erfolg von konventioneller EVAR bzw. fenestrierter oder gebranchter EVAR (F/B-EVAR) und chimney EVAR (CH-EVAR) entscheiden. Der digitale Zwilling kann daher einerseits als prädiktives Werkzeug für präzise Vorhersagen in der präoperativen Planungsphase verwendet werden und andererseits auch parallel zum realen Zwilling existieren mit dem Vorteil, dass zusätzliche Kenngrößen, wie z. B. Spannungszustände im Bereich der Stentgraft Landungs- und Konnektionszonen, ermittelt werden können. Durch die Verbindung von realem und digitalem Zwilling, regelmäßigem Datentransfer sowie Datenabgleich und Anpassung des Models des digitalen Zwillings kann der digitale Zwilling eine äußerst wirklichkeitsgetreue Abbildung des realen Zwillings darstellen.

Der bereits oben angedeutete häufig erfahrungsbasierte Entscheidungsprozess in der präoperativen Planung kann durch den objektiven Charakter des digitalen Zwillings in einen objektiveren präoperativen Planungsprozess umgestaltet werden. Die folgenden drei Anwendungen eines digitalen Zwillings in der endovaskulären AAA Versorgung werden in diesem Beitrag exemplarisch demonstriert:

- Prädiktive Einschätzung der Wahrscheinlichkeit von Komplikationen basierend auf mechanischen und geometrischen Kenngrößen.

- Objektivierung und Optimierung der Stentgraft Auswahl aus dem Portfolio der marktgängigen Stentprothesensysteme

- Bestimmung des Stentgraft Oversizings, d. h. Auswahl des optimalen Stentgraft Durchmessers für den einzelnen Patienten.

Die oben angedeutete Stentgraft Auswahl kann dabei z. B. hinsichtlich Platzierbarkeit Radialkraft, supra-. oder infrarenaler Fixierung, Flexibilität, Festigkeit der Stengraftkomponentenverbindungen und Material (ePTFE vs. Polyester) erfolgen. Darüber hinaus könnten mit einem digitalen Zwilling insbesondere bei komplexen AAA Geometrien unterschiedliche Versorgungsvarianten wie z. B. F-EVAR oder CH-EVAR bezüglich Ihrer Machbarkeit und Langzeitergebnisse bereits vor Implantation verglichen werden. Weitere Anwendungen könnten in der ärztlichen Ausbildung als simulationsbasiertes Trainingstool oder später in der Planung und Herstellung eines echten patientenspezifischen Stentgrafts durch z. B. Rapid Prototyping liegen. Eine vorherige klinische Evaluation durch zunächst monozentrische klinische Studien ist hier natürlich obligat.

\section{Review von Simulationen in der endovaskulären AAA Versorgung}

Abgesehen von Simulation der Hämodynamik der postoperativen Konfiguration (z. B. [6]), lässt sich die Literatur zu Simulationen in der endovaskulären AAA Versorgung grob in die folgenden beiden Kategorien einteilen:

\section{Simulationsmodelle für Stentgrafts und AAAs}

Für die Erstellung eines digitalen Zwillings als prädiktives Werkzeug in der endovaskulären AAA Versorgung ist sowohl ein wirklichkeitsgetreues Modell des Stentgrafts als auch ein wirklichkeitsgetreues Modell des präoperativen AAA unabdingbar. Wirklichkeitsgetreue Stentgraft Modelle wurden z. B von Demanget et al. [3] und Roy et al. [16] entwickelt und experimentell validiert. Zahlreiche Studien zu 
wirklichkeitsgetreuen AAA Modellen existieren, wobei das Ziel der Studien meist die Abschätzung des AAA Rupturrisikos basierend auf AAA Wandspannungen und AAA Wandfestigkeiten ist (z. B. [11][15]).

\section{Virtuelle endovaskuläre Aneurysmenreparatur}

Ziel einer virtuellen endovaskulären AAA Reparatur ist meist die Vorhersage der postoperativen Konfiguration von Stentgraft und AAA basierend ausschließlich auf präoperativen Daten. Die wirklichkeitsgetreue Abbildung der intraoperativen Schritte ist dabei meist nicht von Bedeutung. Außer der Stentgraft Prothese werden dabei in der Regel keine weiteren medizinischen Werkzeuge, wie z. B. intraoperative Führungsdrähte, berücksichtigt. In den letzten Jahren wurden zahlreiche Studien zu virtuellen endovaskulären Aneurysmenreparaturen in idealisierten Geometrien veröffentlicht (z. B. [8][14]). Aufgrund der hohen Komplexität ist die Anwendung in patientenspezifischen Geometrien jedoch auf wenige Studien limitiert [7][1][12][13][4]. Basierend auf der Methodik der Stentgraft Platzierung und des Stentgraft Deployments, lassen sich die Studien zu virtuellen endovaskulären Aneurysmenreparaturen grob in drei Kategorien einteilen. Zunächst adaptierte Auricchio et al. [1] die Methodik von Simulationen reiner Stents (d. h. ohne Graftmantel), wobei ein virtueller Katheter als Hilfswerkzeug zur Stentgraft Platzierung und zum Stentgraft Deployment verwendet wird. Perrin et al. [12] entwickelten eine völlig neue Methodik zur virtuellen endovaskulären Aneurysmenreparatur, welche vollständig auf die Modellierung von Hilfswerkzeugen wie dem virtuellen Katheter verzichten kann. Bei dieser Methodik wird ein Morphing Algorithmus verwendet welcher das AAA und den Stentgraft, der sich innerhalb des AAA befindet, in die postoperative Konfiguration verformt. Kürzlich wurde eine weitere Methodik zur virtuellen endovaskulären Aneurysmenreparatur von unserer Gruppe veröffentlicht [8][7]. Dabei wird der Stentgraft mithilfe eines Morphing Algorithmus vollautomatisch in dem patientenspezifischen AAA platziert ohne dass die Modellierung weiterer Hilfswerkzeuge nötig ist.

\section{Prädiktive Komplikationseinschätzung für klinische Fälle}

\section{Prozesskette für die prädiktive Komplikationseinschätzung der endovaskulären AAA Versorgung}

Für die prädiktive Komplikationseinschätzung klinischer Fälle wird eine durchgängige und weitestgehend vollautomatisierte Prozesskette durchlaufen, welche in Abbildung 1 dargestellt ist. 


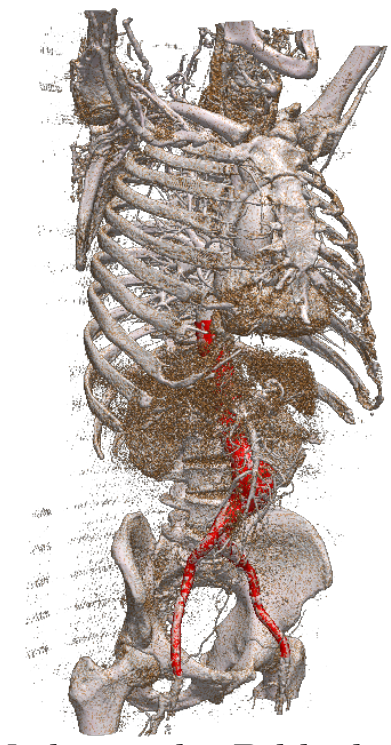

Medizinische Bildgebung

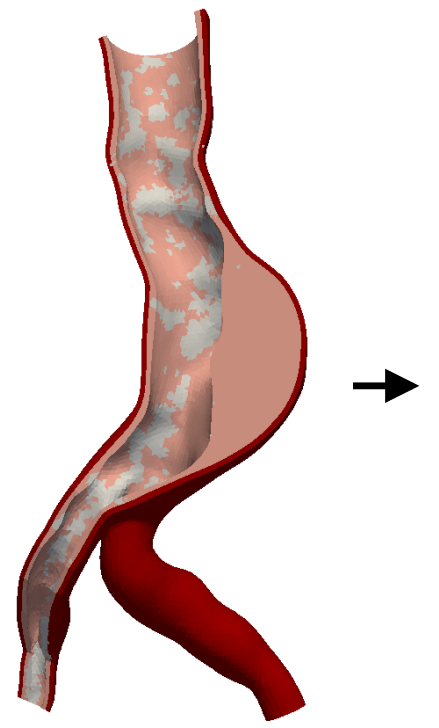

Modellerstellung

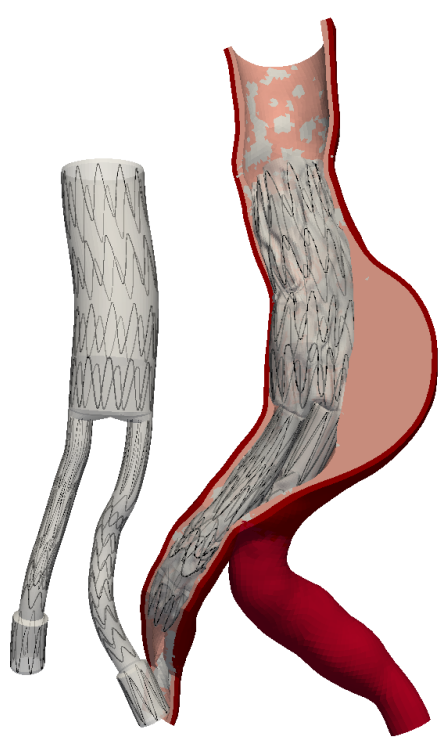

Simulation

Abbildung 1: Prozesskette für die prädiktive Komplikationseinschätzung in der endovaskulären AAA Versorgung.

Zunächst wird die patientenspezifische AAA Geometrie einschließlich intraluminalem Thrombus und Kalkablagerungen halbautomatisch unter Verwendung kommerzieller Segmentierungssoftware aus präoperative CT Daten segmentiert. Anschließend wird aus der segmentierten AAA Geometrie vollautomatisch ein wirklichkeitsgetreues AAA Simulationsmodell erstellt, welches u.a. Aortenwand, intraluminalen Thrombus, Kalkablagerungen, kohortengemittelte Materialgesetze für diese Bestandteile und einen physiologischen Blutdruck berücksichtigt. Zusätzlich wird durch Spezifikation eines Stentgraft Typs und einer Stentgraft Größe vollautomatisch ein Stentgraft Modell erzeugt. Anschließend wird durch die virtuelle endovaskuläre AAA Reparatur die postoperative AAA und Stentgraft Konfiguration berechnet.

Basierend auf der berechneten postoperativen AAA und Stentgraft Konfiguration kann die Komplikationswahrscheinlichkeit nach der endovaskulären AAA Reparatur abgeschätzt werden. Dafür werden geometrische und mechanische Kenngrößen verwendet, wie z. B. Stentgraft induzierte Überspannungen in der Aortenwand oder der Abstand zwischen Stentgraft und Aorta in den Stentgraft Landungszonen. Stentgraft induzierte Überspannungen kennzeichnen eine Überbelastung der umliegenden Aortenwand. Der Abstand zwischen Stentgraft und Aorta ist ein Maß für die Qualität der Abdichtung zwischen Stentgraft und Gefäßwand in den Stentgraft Landungszonen. Ein großer Abstand zwischen Stentgraft und Aorta in den Stentgraft Landungszonen erhöht die Wahrscheinlichkeit einer Endoleckage Typ I.

\section{Validierung des digitalen Zwillings mit postoperativen CT Daten}

Zur Validierung und Genauigkeitsabschätzung der Approximation durch den digitalen Zwilling wird die postoperative Konfiguration des digitalen Zwillings mit postoperativen CT Daten wenige Tage nach der endovaskulären AAA Reparatur verglichen (Abb. 2). Qualitativ unterscheiden sich die postoperativen Konfigurationen des digitalen Zwillings und des realen Zwillings (postoperative CT Daten) kaum. Quantitativ ergibt sich ein relativer Fehler in der Approximation des postoperativen Stentgraft Durchmessers von 
$5.6 \pm 8.1 \% \quad$ (Mittelwert +/- Standardabweichung) über alle untersuchten patientenspezifischen Fälle [7].

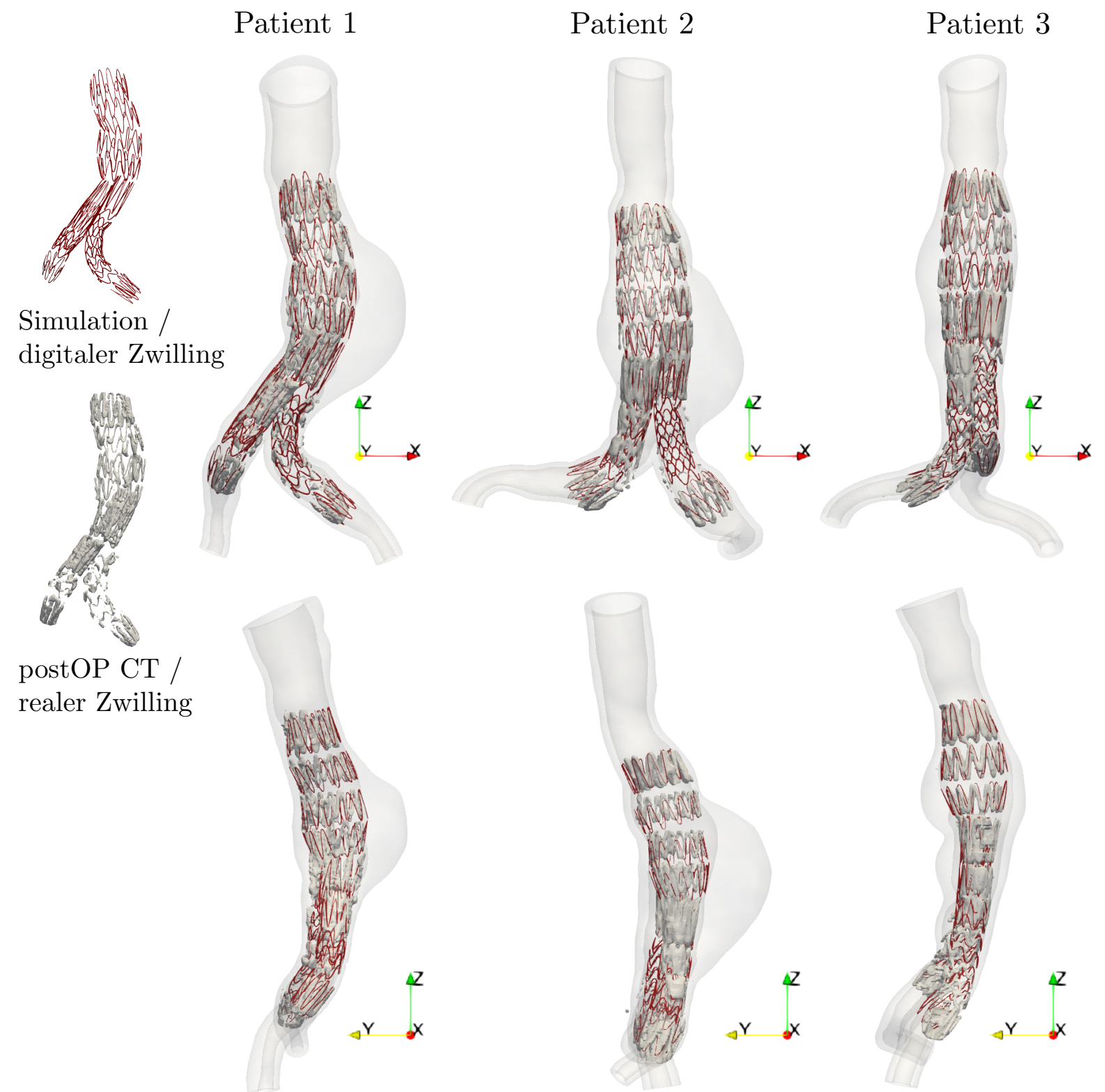

Abbildung 2: Validierung des digitalen Zwillings mit postoperativen CT Daten. In den Abbildungen werden die Stents von digitalem und realem Zwilling gemeinsam abgebildet um die Übereinstimmung zu visualisieren.

\section{Überspannungen in den Stentgraft Landungszonen}

Im Gegensatz zum realen Zwilling lassen sich am digitalen Zwilling auch mechanische Kenngrößen ermitteln, die rein mithilfe medizinischer Bildgebung nicht bestimmbar sind. In Abbildung 3 sind exemplarisch die Wandspannungen vor und nach der endovaskulären AAA Reparatur sowie die Überspannungen in der Aortenwand für vier patientenspezifische Fälle präsentiert. Positive Überspannungen kennzeichnen Bereiche der Aortenwand in welchen die postoperativen Wandspannungen größer als die präoperativen Wandspannungen sind. Negative Überspannungen kennzeichnen Bereiche in welchen die Wandspannungen vor der endovaskulären AAA Reparatur größer sind als die Wandspannungen nach der endovaskulären AAA Reparatur. Durch das Einsetzen des Stentgrafts werden im Modell die Wandspannungen im Aneurysmasack in Patient 2, 3 und 4 deutlich verringert. Bei Patient 1 ist der luminale 
Durchmesser im Aneurysmasack aufgrund einer ausgeprägten Thrombus Schicht relativ klein sodass der Stentgraft fast vollständig mit dem Thrombus im Aneurysmasack in Kontakt ist. Daher nehmen die Wandspannungen im Aneurysmasack im digitalen Zwilling von Patient 1 nach der endovaskulären AAA Reparatur nicht ab.

Trotz der meist erfolgreichen Reduzierung der Wandspannungen im Aneurysmasack kommt es im Bereich der proximalen und distalen Landungszonen des Stentgrafts zu einer erheblichen Zunahme der Wandspannungen auf über $300 \mathrm{kPa}$. Diese Überspannungen werden häufig als möglicher Grund für Langzeitkomplikationen nach EVAR, wie z. B. die sekundäre Halsdilatation, genannt [2].

Der Spannungszustand in der Aortenwand ist nur eine von vielen mechanischen Kenngrößen die sich durch den digitalen Zwilling vorhersagen lassen. Für einen detaillierten Überblick an geometrischen und mechanischen Kenngrößen zur prädiktiven Komplikationseinschätzung sei auf [9] verwiesen.

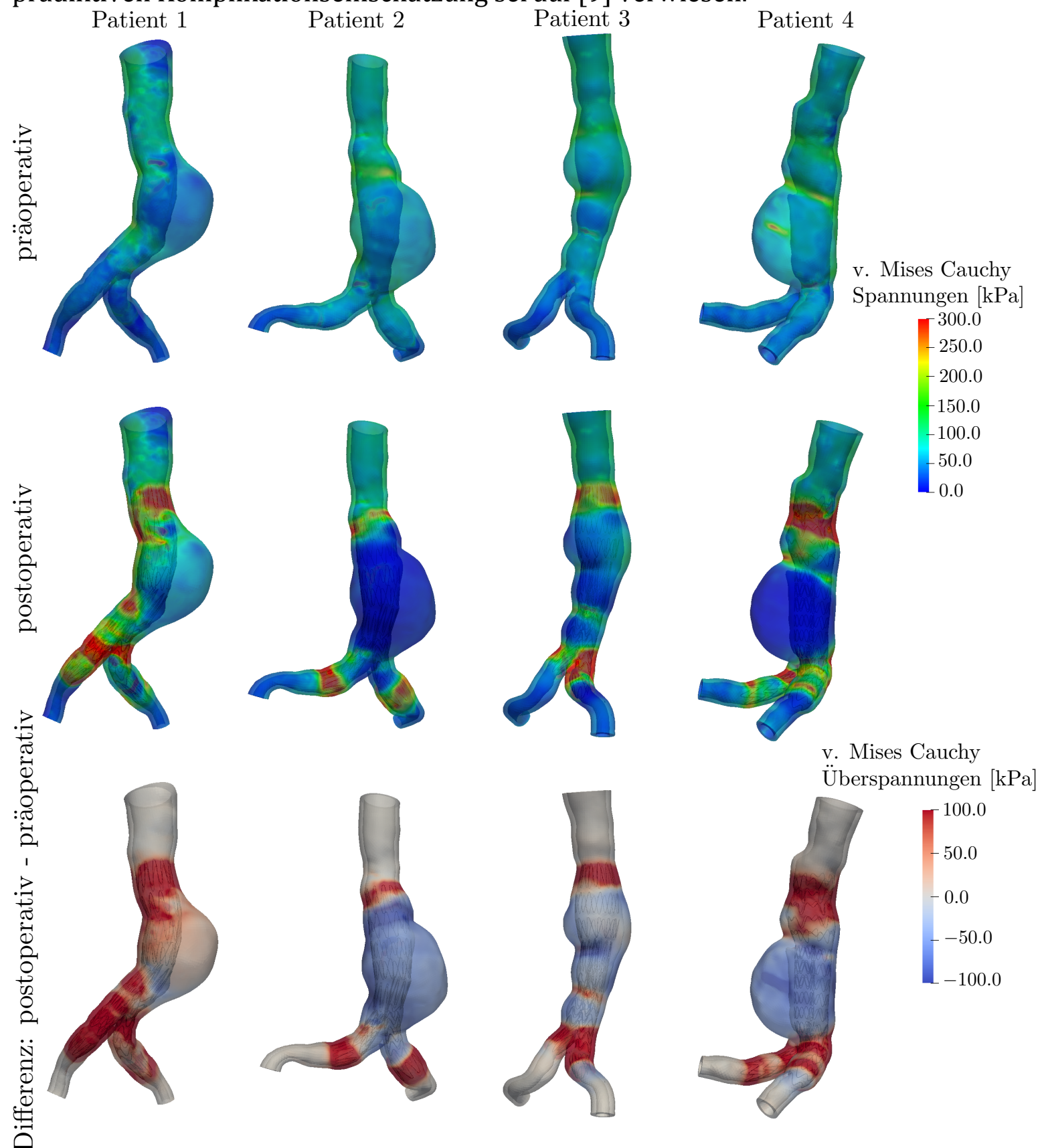

Abbildung 3: Präoperative und postoperative Wandspannungen sowie Stentgraft induzierte Überspannungen in der Aortenwand unter einem systolischem Blutdruck von $130 \mathrm{mmHg}$. 


\section{Stentgraft Auswahl und Oversizing am digitalen Zwilling}

\section{Einfluss der AAA Geometrie und des Stentgraft Designs}

Sowohl die AAA Geometrie als auch das Stentgraft Design können einen entscheidenden Einfluss auf die Qualität der endovaskulären AAA Reparatur haben. Diese beiden Einflüsse sind in Abb. 4 exemplarisch für die Variation des Winkels des AAA Halses (Abb. 4I) und die Variation der Stentringhöhe (Abb. 4II) dargestellt.

Je größer der Winkel des AAA Halses, desto mehr resultiert der arterielle Blutdruck als integrale Kraft $F_{\text {drag }}$ die auf die Stentgraft Prothese wirkt. Während bei einer geraden Aorta (Winkel des AAA Halses $\alpha_{p}=0^{\circ}$ ) die resultierenden hydrostatischen BlutdruckKräfte null sind, ergeben sich für einen Winkel des AAA Halses von $\alpha_{p}=80^{\circ}$ unter einem systolischem Blutdruck von 130 mmHg Kräfte von $8.7 \mathrm{~N}$ für den betrachteten Fall. Es sei angemerkt, dass aufgrund des geraden distalen Endes der Aorta, im gegebenen Beispiel die resultierende Kraft stets in proximal-laterale Richtung zeigt. Bei einem nicht geraden distalen Ende der Aorta kann die resultierende Kraft ebenfalls in distale Richtung orientiert sein.

Abb. 2II verdeutlicht den Einfluss von Stentgraft Design Parametern auf die Qualität der endovaskulären AAA Reparatur am Beispiel der Variation der Stentringhöhe. Je größer die Stentringhöhe $h^{S}$, desto weniger flexibel ist die Stentgraft Prothese. Die Wahrscheinlichkeit eines Knickes der Prothese mit den damit verbundenen Komplikationen, wie z. B. Prothesenverschlüsse, nimmt zu.

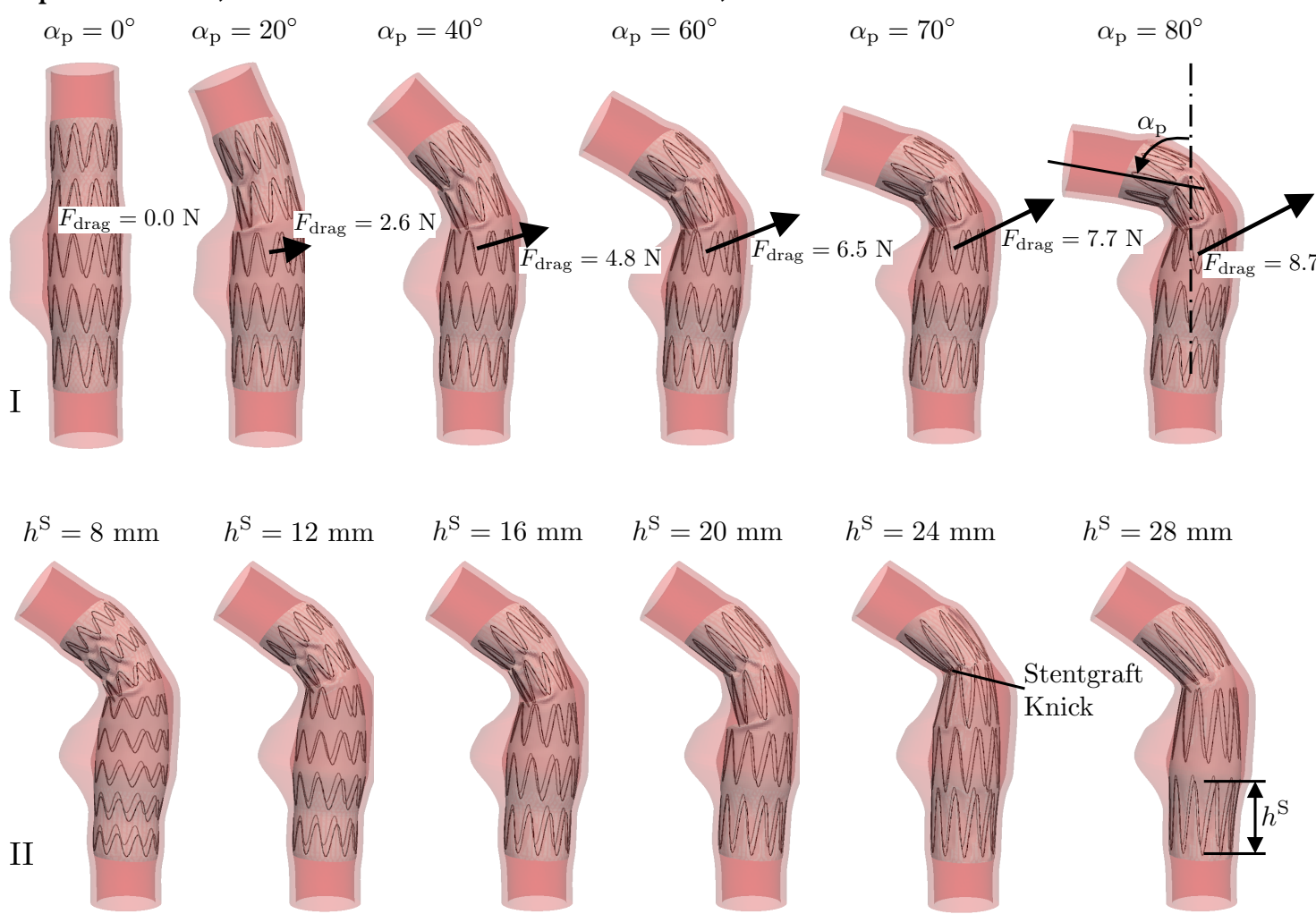

Abbildung 4: Einfluss des Winkels des AAA Halses auf die resultierende hydrostatische Blutdruck-Kraft (I) und Einfluss des Stentgraft Designs auf die Flexibilität des Stentgrafts (II).

\section{Einfluss des Stentgraft Oversizings}

Um eine ordnungsgemäße Fixierung des Stentgrafts und eine leckagefreie Abdichtung zwischen Stentgraft und Aorta in den Stentgraft Landezonen zu erreichen, muss der 
Stentgraft im Bezug auf den Aortendurchmesser überdimensioniert sein. Die meisten Stentgraft Hersteller empfehlen ein Oversizing des Stentgrafts zwischen 10\% und 20\%. Jedoch ist das ideale Oversizing für individuelle patientenspezifische Fälle schwierig abzuschätzen. Am digitalen Zwilling können in der präoperativen Planung verschiedene Stentgraft Oversizings verglichen („durchprobiert“) werden und das individuell ideale Oversizing ermittelt werden.

Abb. 5 zeigt den Einfluss des Stentgraft Oversizing $o$ auf die Stentgraft Fixierungskraft. Oberhalb eines kritischen Oversizing $o_{\text {crit }}$ nimmt die Stentgraft Fixierungskraft nicht weiter zu. Für den dargestellten Fall hat demzufolge ein Oversizing des Stentgrafts von mehr als 15\% im diastolischen und mehr als 20\% im systolischen Zustand keinen weiteren positiven Effekt auf die Stentgraft Fixierungskraft. Der Wert des kritischen Oversizing $o_{\text {crit }}$ hängt jedoch stark von dem zugrundeliegenden Blutdruck sowie von der Geometrie des AAA und des Stentgrafts ab. Das Plateau in der Stentgraft Fixierungskraft oberhalb des kritischen Oversizing $o_{\text {crit }}$ lässt sich mechanisch durch das zunehmende Beulen des Stentgrafts und einer verringerten Kraftübertragung von Stentgraft auf die Aorta erklären. Im diastolischen Zustand von $80 \mathrm{mmHg}$ (gestrichelte Linie) nimmt bei dem dargestellten Beispiel das radiale Beulen des Stentgrafts ab einem Stentgraft Oversizing von ca. 15\% drastisch zu. Im systolischen Zustand von $130 \mathrm{mmHg}$ (durchgezogene Linie) presst der höhere Blutdruck die Stentgraft Prothese stärker gegen die luminale Aortenoberfläche. Das radiale Beulen des Stentgrafts nimmt daher im systolischen Zustand erst ab einem Oversizing von ca. $20 \%$ drastisch zu.

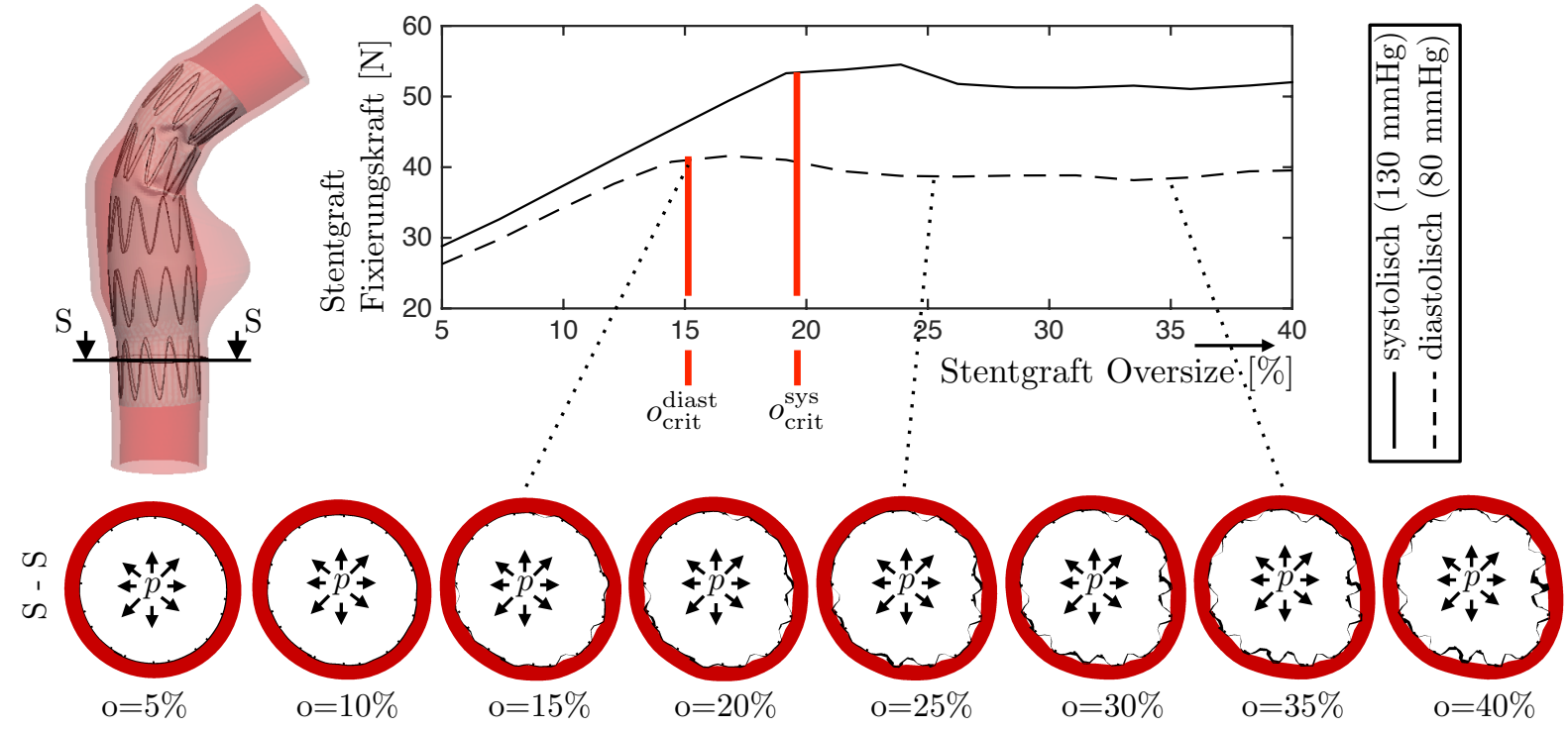

Abbildung 5: Einfluss des Stentgraft Oversizing auf die Stentgraft Fixierungskraft.

\section{Klinisches Beispiel: Stentgraft Auswahl}

Der behandelnde Arzt hat in der Regel Stentgrafts unterschiedlicher Hersteller und unterschiedlicher Größen zur Auswahl, wobei die endgültige Auswahl des Stentgrafts aktuell zu einem großen Teil eine subjektive Entscheidung des Arztes ist.

Für ein möglichst reales Setting werden zwei Stentgrafts A und B von unterschiedlichen Herstellern in einem patientenspezifischen Fall mithilfe des digitalen Zwillings verglichen. Basierend auf den IFU des entsprechenden Herstellers, wird für den vorliegenden Patienten mit mittlerem Aneurysmenhalsdurchmesser von $25.5 \mathrm{~mm}$ von Hersteller A ein Stentgraft mit einem proximalen Durchmesser von $30 \mathrm{~mm}$ und von Hersteller B ein Stentgraft mit einem proximalen Durchmesser von $32 \mathrm{~mm}$ empfohlen. Der behandelnde Arzt steht nun vor der Entscheidung welche der beiden Prothesen für 
den vorliegenden Patienten die bessere Lösung darstellt. Diese meist erfahrungsbasierte Entscheidungsfindung kann mithilfe der Verwendung eines digitalen Zwilling objektiviert und optimiert werden.

Exemplarisch wird nur die Qualität der Abdichtung in der proximalen Landungszone in Abbildung 6 verglichen. Wie bereits in Kapitel 3 erwähnt, können noch zahlreiche weitere geometrische und mechanische Kenngrößen am digitalen Zwilling zur Abschätzung der Qualität des platzierten Stentgrafts verwendet werden. Während Stentgraft A zu einem leichten Stent Kollaps in der proximalen Landungszone neigt, ergibt sich für Stentgraft B eine sehr gute und gleichmäßige Abdichtung in der proximalen Landungszone. Trotz des größeren Oversizing neigt aufgrund des unterschiedlichen Designs der Stent Ringe der beiden Stentgraftprothesen, Stentgraft A eher zu einem Stent Kollaps und den damit verbundenen relativ großen Klaffungen zwischen Stentgraft und Aorta. Die Wahrscheinlichkeit einer Endoleckage Typ 1 könnte damit bei Stentgraft A größer als bei Stentgraft B sein.

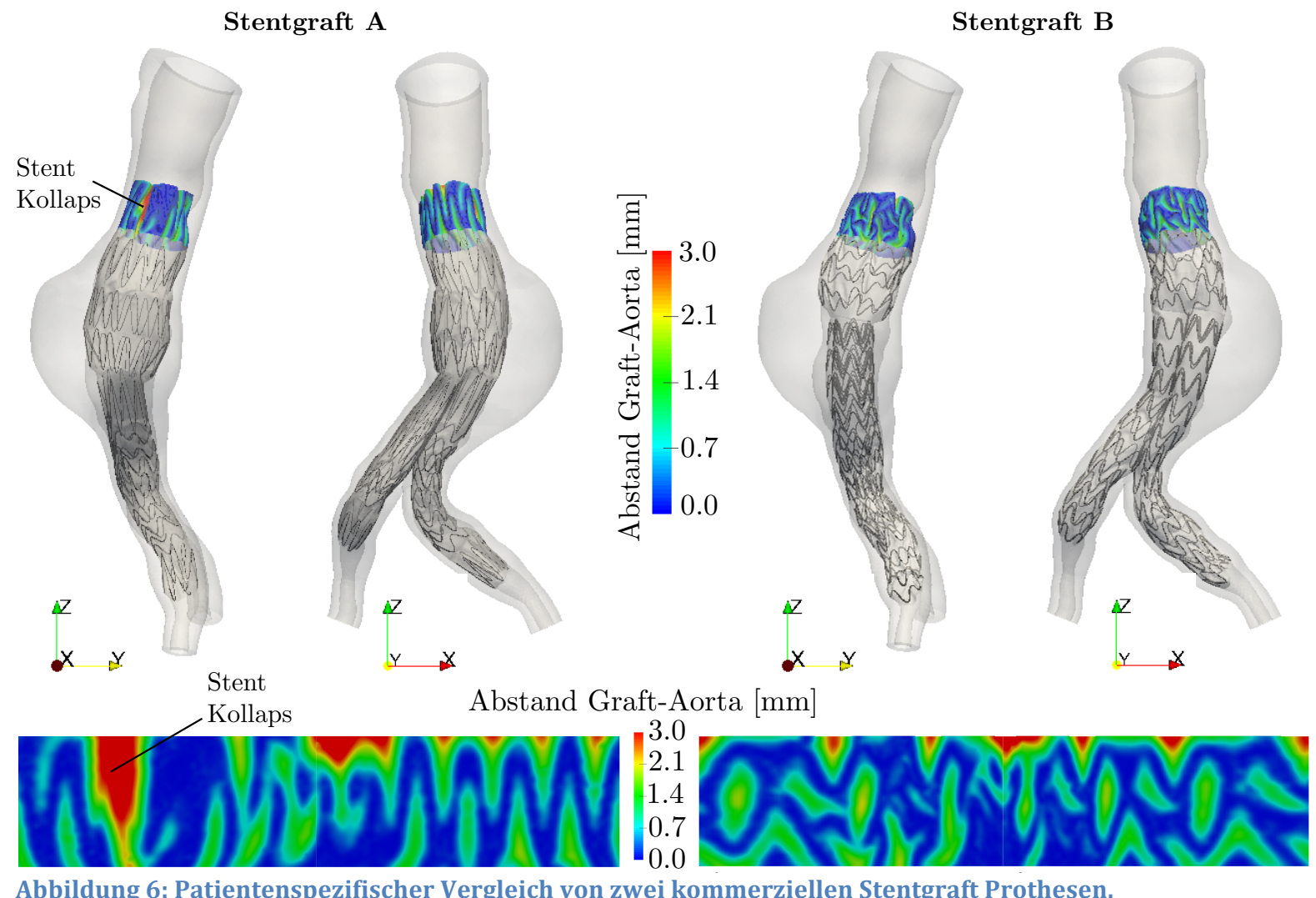

\section{Fazit und Zukunftsperspektive}

Die gute Übereinstimmung zwischen digitalem und realem Zwilling macht den digitalen Zwilling $\mathrm{zu}$ einem vielsprechenden Planungswerkzeug in der präoperativen Planungsphase der endovaskulären AAA Versorgung. Der digitale Zwilling kann dabei in der klinischen Anwendung zur besseren Auswahl und Größenbestimmung des Stentgrafts beitragen und damit die Komplikationsrate der endovaskulären AAA Reparatur reduzieren. Weiterhin kann der digitale Zwilling zur Weiterentwicklung von Stentgraft Prothesen und der Weiterentwicklung des EVAR Prozesses ein wertvolles Werkzeug in der klinischen Forschung darstellen. 
Weitere notwendige Schritte auf dem Weg zur klinischen Anwendung des digitalen Zwillings sind u.a. die Reduzierung der benötigten Rechenleistung, die vollständige Automatisierung der Prozesskette sowie die Validierung des digitalen Zwillings an einer für medizinische Langzeitstudien angemessen großen Studienkohorte.

\section{Acknowledgments}

AH und MWG danken der Unterstützung dieser Arbeit durch das Leibniz Rechenzentrum der Bayerischen Akademie der Wissenschaften (LRZ) unter pr48ta sowie der DFG unter GE2254/4-1.

\section{Literatur}

1. Auricchio F, Conti M, Marconi S et al (2013) Patient-specific aortic endografting simulation: From diagnosis to prediction. Comput Biol Med 43:386-394.

2. Corbett TJ, Callanan A, Morris LG et al (2008) A review of the in vivo and in vitro biomechanical behavior and performance of postoperative abdominal aortic aneurysms and implanted stent-grafts. J Endovasc Ther 15:468-484.

3. Demanget N, Duprey A, Badel P et al (2013) Finite element analysis of the mechanical performances of 8 marketed aortic stent-grafts. J Endovasc Ther 20:523535.

4. Derycke L, Perrin D, Cochennec F et al (2019) Predictive Numerical Simulations of Double Branch Stent-Graft Deployment in an Aortic Arch Aneurysm. Ann Biomed Eng 1-12.

5. El Saddik A (2018) Digital twins: the convergence of multimedia technologies. IEEE Multimed 25:87-92.

6. Figueroa CA, Taylor CA, Yeh V et al (2009) Effect of curvature on displacement forces acting on aortic endografts: a 3-dimensional computational analysis. J Endovasc Ther 16:284-294.

7. Hemmler A, Lutz B, Kalender G et al (2019) Patient-specific in silico endovascular repair of abdominal aortic aneurysms: application and validation. Biomech Model Mechanobiol 18:983-1004.

8. Hemmler A, Lutz B, Reeps C et al (2018) A methodology for in silico endovascular repair of abdominal aortic aneurysms. Biomech Model Mechanobiol 17:1139-1164.

9. Hemmler A, Lutz B, Reeps C, Gee MW (2019) In silico study of vessel and stentgraft parameters on the potential success of endovascular aneurysm repair, ahead of print, https://doi.org/10.1002/cnm.3237

10. Kühnl A, Erk A, Trenner M et al (2017) Incidence, Treatment and Mortality in Patients with Abdominal Aortic Aneurysms: An Analysis of Hospital Discharge Data from 2005--2014. Dtsch Ärztebl Int 114:729-736.

11. Maier A, Gee MW, Reeps C et al (2010) A comparison of diameter, wall stress, and rupture potential index for abdominal aortic aneurysm rupture risk prediction. Ann Biomed Eng 38:3124-3134.

12. Perrin D, Badel P, Orgéas L et al (2015) Patient-specific numerical simulation of stent-graft deployment: Validation on three clinical cases. J Biomech 48:1868-1875.

13. Perrin D, Badel P, Orgeas L et al (2016) Patient-specific simulation of endovascular repair surgery with tortuous aneurysms requiring flexible stent-grafts. J Mech Behav Biomed Mater 63:86-99. 
14. Perrin D, Demanget N, Badel P et al (2015) Deployment of stent grafts in curved aneurysmal arteries: toward a predictive numerical tool. Int J Numer Methods Biomed Eng 31:e02698.

15. Polzer S, Gasser TC (2015) Biomechanical rupture risk assessment of abdominal aortic aneurysms based on a novel probabilistic rupture risk index. J R Soc Interface 12:20150852.

16. Roy D, Lerouge S, Inaekyan K et al (2016) Experimental validation of more realistic computer models for stent-graft repair of abdominal aortic aneurysms, including pre-load assessment. Int J Numer Methods Biomed Eng 32:e02769.

17. Schanzer A, Greenberg RK, Hevelone N et al (2011) Predictors of Abdominal Aortic Aneurysm Sac Enlargement After Endovascular Repair. Circulation 123:28482855. 